International Journal of Graph Computing

Vol. 1, No. 1 (2020) 1-24

(C) KS Press, Institute for Semantic Computing Foundation

DOI: $10.35708 / \mathrm{GC} 1868-126721$

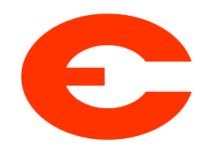

\title{
The Leaf Function of Graphs Associated with Penrose Tilings
}

\author{
Carole Porrier and Alexandre Blondin Massé \\ Laboratoire de Combinatoire et d'Informatique Mathématique (LaCIM) \\ Université du Québec à Montréal \\ Montréal (Québec), Canada \\ porrier.carole@uqam.ca
}

Received (11/02/2019)

Revised $(01 / 19 / 2020)$

Accepted $(02 / 28 / 2020)$

\begin{abstract}
In graph theory, the question of fully leafed induced subtrees has recently been investigated by Blondin Massé et al. in regular tilings of the Euclidian plane and 3-dimensional space. The function $L_{G}$ that gives the maximum number of leaves of an induced subtree of a graph $G$ of order $n$, for any $n \in \mathbb{N}$, is called leaf function. This article is a first attempt at studying this problem in non-regular tilings, more specifically Penrose tilings. We rely not only on geometric properties of Penrose tilings, that allow us to find an upper bound for the leaf function in these tilings, but also on their links to the Fibonacci word, which give us a lower bound. In particular, we show that $2 \varphi n /(4 \varphi+1) \leq L_{k d}(n) \leq\lfloor n / 2\rfloor+1$, for any $n \in \mathbb{N}$, where $\varphi$ is the golden ratio and $L_{k d}$ is the leaf function for kites and darts Penrose tilings. Our approach rely on a purely discrete representation of points in the tilings, thus preventing numerical errors and improving computation efficiency. Finally, we present a procedure to dynamically generate induced subtrees without having to generate the whole patch surrounding them.
\end{abstract}

Keywords: Fully leafed induced subtrees, Leaf function, Graph computing, Penrose tilings, Discrete representation, Ammann bars, Fibonacci word

\section{Introduction}

Since Roger Penrose introduced them in 1974 [12 and described them with more details in [13] and [14], Penrose tilings have aroused great interest among many mathematicians, including Robert Ammann and Raphael M. Robinson whose tilings of the plane are also quite interesting (see [10], Sections 10.2 and 
10.4). Martin Gardner, known for his popularization of mathematics by proposing recreational puzzles in written media, greatly contributed to making these tilings famous [8], and we owe much of the vocabulary specific to Penrose tilings as well as many of their known properties to John H. Conway. Penrose tilings are so remarkable that Grünbaum and Shephard devoted several sections of their book on tilings to the study of Penrose tilings based on the work of these four researchers and Roger Penrose himself, including unpublished notes and private communications 910. Furthermore, the algebraic study of Penrose tilings by de Bruijn [6] allowed for the discovery of quasicrystals, that is crystals whose structure is not periodic [17.

Initially, Penrose described three interconnected sets of tiles, called respectively P1, P2 and P3. The set P1 is composed of six prototiles, while the sets $\mathrm{P} 2$ and $\mathrm{P} 3$ both use only 2 prototiles. The tiles of $\mathrm{P} 2$ are called kites and darts and an example of a tiling by P2 is illustrated in Figure 1. The two tiles of P3 are called rhombs, one being thinner and the other thicker. Since those tilings all share similar properties [10] but still differ enough to be studied separately, we shall only focus on "kites and darts" tilings in the remaining part of this article.

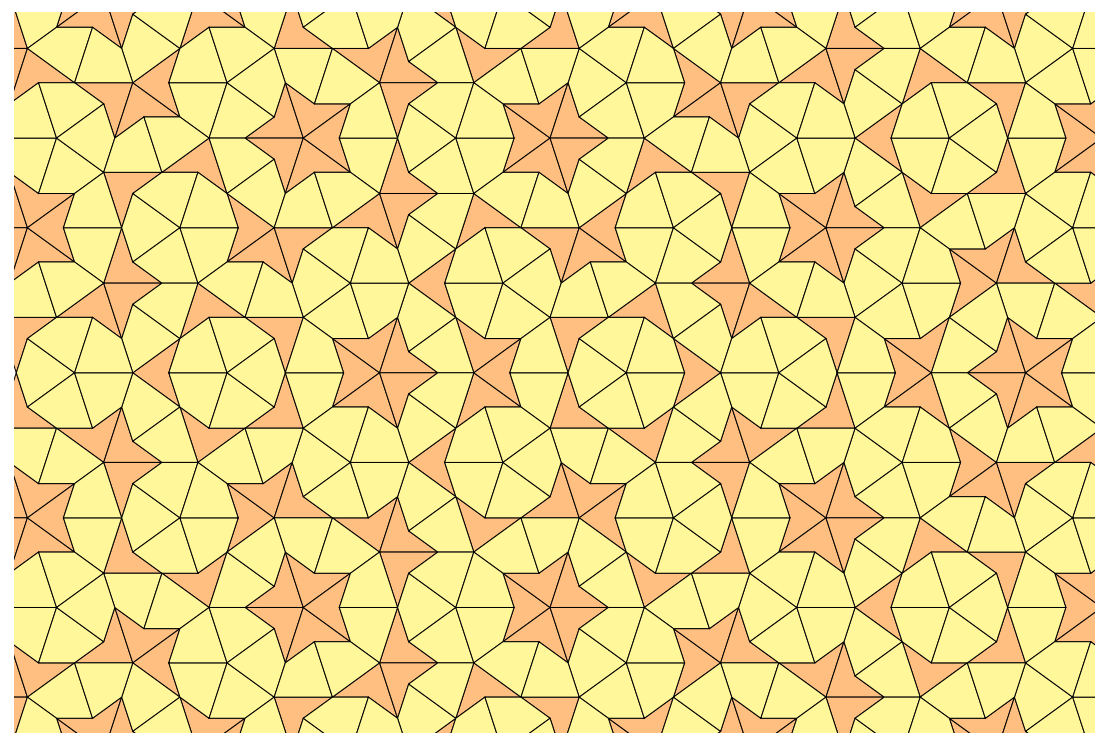

Fig. 1: A Penrose tiling by kites and darts.

Although they are intrinsically geometric objects, Penrose tilings can be viewed as simple graphs, whose vertices are the tiles and whose adjacency relation in the graph is the adjacency relation between tiles in the tiling. In particular, one might be interested in inspecting its subgraphs in order to gain some insight about its global structure. In a recent series of publications, Blondin Massé and his co-authors have studied the fully leafed induced subtrees, i.e. subtrees that 
maximize the number of leaves with respect to their order, appearing in different families of graphs ([45|2]). They looked at the four basic periodic lattices: the square, the triangle and the hexagonal lattices in $2 \mathrm{D}$, and the cubic lattice in $3 \mathrm{D}$. One of their results consisted in describing the leaf function $L$ in each of these four lattices, i.e. the map associating with each integer $n \geq 2$ the number of leaves of a fully leafed induced subtree of $n$ vertices 5 .

This article aims to provide similar results for Penrose tilings, a first step in studying non-periodic tilings, where the situation is more intricate. Although we are not sure whether the induced subtrees built in Section 5 are fully leafed, they present remarkable properties and are closely linked to the Fibonacci word. Another interesting observation is that they are caterpillar graphs, i.e. trees such that the removal of all their leaves yields a chain graph. In particular, since they have a linear structure, they might be interpreted as words embedded in graphs. For instance, in [3], the second author of this article and his co-authors established a link between the leaf function of caterpillar graphs and prefixnormal words, a family of words closely related to the bubble sort [7].

The remaining sections are divided as follows. In Section 2, we present the basic definitions and notations on tilings, graphs and words, and the known properties of "kites and darts" Penrose tilings. Our first computational experiments and their limitations are summarized in Section 3. Using geometrical properties, Section 4 then presents an upper bound for their leaf function, while in Section 5 we exhibit special caterpillars having many leaves, therefore providing a lower bound for the leaf function. Section 6 details the computational aspects and representation of Penrose tilings. Finally, Section 7 is devoted to the dynamic generation of Penrose patches and Penrose trees.

Note that Sections 1-2 and 4-6 of this article were already in an earlier version of the paper, originally presented at IEEE Graph Computing 2019 [15. In this extended version, we provide more details about the computational aspects in Sections 3 , 6 and 7

\section{Preliminaries}

We now recall some useful definitions and notations about tilings, graphs and words, before saying more about Penrose tilings.

\subsection{Tilings, graphs and words}

A tiling of the plane is a countable family $\mathcal{T}=\left\{T_{n}\right\}_{n \in \mathbb{N}}$ of closed sets of $\mathbb{R}^{2}$ which is both a covering and a packing of the plane, that is (1) tiles $T_{1}, T_{2}, \ldots$ verify $\bigcup_{n \in \mathbb{N}} T_{n}=\mathbb{R}^{2}$ and (2) for all $i \neq j$, $\operatorname{int}\left(T_{i}\right) \cap \operatorname{int}\left(T_{j}\right)=\emptyset$. The intersection of any two tiles is either a set of edges (line segments) and/or vertices (points), or the empty set. Two tiles are called adjacent if they share an edge. Two tilings $\mathcal{T}_{1}$ and $\mathcal{T}_{2}$ are congruent if there is an isometry $\sigma$ of the plane such that $\mathcal{T}_{1}=\sigma\left(\mathcal{T}_{2}\right)$. We say that two tilings $\mathcal{T}_{1}$ and $\mathcal{T}_{2}$ are equal if there is a similarity $\tau$ of the plane (i.e. an isometry followed by a scaling) such that $\mathcal{T}_{1}=\tau\left(\mathcal{T}_{2}\right)$. A tiling $\mathcal{T}$ is called monohedral if any tile of $\mathcal{T}$ is congruent with a given set $T$, that is if all tiles have the same size and shape. This set $T$ is called prototile and we say that 
the prototile $T$ admits the tiling $\mathcal{T}$. A tiling $\mathcal{T}$ is dihedral if any of its tiles $T_{i}$ is congruent with any of two (non-congruent) prototiles $T$ and $T^{\prime}$. This article focuses on Penrose tilings which are dihedral, where the prototiles are a kite and a dart.

If $\mathcal{T}$ is a tiling, an isometry $\sigma$ is a symmetry of $\mathcal{T}$ if the image through $\sigma$ of any tile of $\mathcal{T}$ is a tile of $\mathcal{T}$, which also preserves markings and colors when there are any. A tiling is periodic if its symmetry group contains at least two translations in non-parallel directions. If a tiling is not periodic but its symmetries include rotations about a fixed point, then this point is unique and is called the center of the tiling. Penrose tilings by kites and darts are all non-periodic but two of them have a center. A patch in a tiling is any set of tiles (not prototiles) whose union forms a connected set without hole. The theory of tilings is extensively described in [10, including the study of Penrose tilings.

The vertices and edges of a tiling form an undirected graph, whose dual is the graph we are interested in. Let $G=(V, E)$ be a simple graph. For any $u \in V$ and $U \subseteq V$, the set of neighbors of $u$ in $G$ is denoted by $N_{G}(u)$, which is naturally extended to $U$ by defining $N_{G}(U)=\left\{N_{G}\left(u^{\prime}\right) \mid u^{\prime} \in U\right\}$. The subgraph of $G$ induced by $U$ is $G[U]=\left(U, E \cap \mathcal{P}_{2}(U)\right)$, where $\mathcal{P}_{2}(U)$ is the set of 2-element subsets of $U$. In this article, the vertices of $G$ are tiles composing a tiling of the plane by Penrose kites and darts, and $E$ is given by the adjacency relation between tiles. The graph thus obtained is infinite but we only consider its finite subgraphs. More precisely, we focus on induced subtrees of fixed order $n$, that is subgraphs of $G$ which are trees, for all $n \in \mathbb{N}$. We denote $\mathcal{T}_{G}(n)$ the set of all

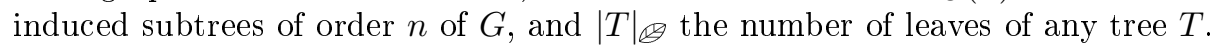
The leaf function of $G$ is then formally defined for all $n \in\{1,2, \cdots,|G|\}$ by

$$
L_{G}(n)=\max \left\{|T|^{\otimes}: T \in \mathcal{T}_{G}(n)\right\} .
$$

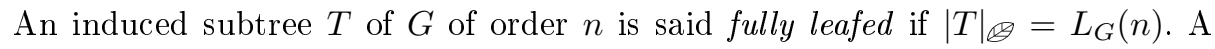
caterpillar graph $C$ is a tree whose derived tree, obtained from $C$ by removing its leaves, is a path [11.

To conclude this subsection, we briefly recall some definitions on words. An alphabet $\mathcal{A}$ is a set of symbols (or characters). A word on $\mathcal{A}$ is a sequence of symbols of $\mathcal{A}$, and the set of all words on $\mathcal{A}$ is denoted $\mathcal{A}^{*}$. We say that a word $y$ is a subword or factor of a word $w$ if there exist words $x, z$ such that $w=x y z$. A binary word is a word on $\{0,1\}$ or any other 2-element alphabet. The Fibonacci word is an infinite binary word, and the structure of Penrose tilings is guided by "musical sequences", that is factors of the Fibonacci word. More information about words can be found in [1, and the properties of musical sequences are quite detailed in [10].

\subsection{Penrose Tilings}

Tilings by kites and darts were first described by Roger Penrose in [13. The dart is actually a non-convex kite and in each tile, the longer side is $\varphi$ times 
longer than the shorter one, where $\varphi=\frac{1+\sqrt{5}}{2}$ is the golden ratio. The tiles must be arranged according to specific assembly rules, for instance using a marking of the tiles, or arrows on the sides, or two colors for the corners of the tiles (opposite corners then have the same color). Following such rules, there are infinitely many ways in which the tiles can be arranged, so that there are infinitely many tilings by Penrose kites and darts, and as many graphs corresponding to them. Most knowledge on Penrose tilings is compiled in [10].

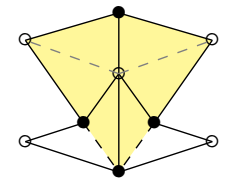

(a) Kite

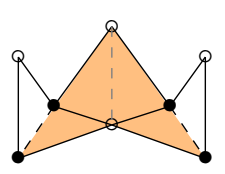

(b) Dart

Fig. 2: Decomposition of kites and darts.

Many properties of these tilings, as well as how to generate them computationally, rely on triangular decomposition: any tile cut along its reflection line gives two isosceles triangles called $A$-tiles - a kite is divided into two large tiles $L_{A}$ and a dart into two short tiles $S_{A}$. A-tiles can in turn be decomposed into smaller tiles of the same shape, with which smaller kites and darts can be recomposed, as shown in Figure 2. Provided a scaling by $\varphi: 1$, the new kites and darts have the same size as the original ones.

There are seven vertex configurations, that is seven ways in which tiles can be arranged around a vertex of the tiling. These are given in Figure 3 . Penrose tilings have the local isomorphism property: any patch of tiles in a tiling by kites and darts (for instance the vertex configurations) is congruent to infinitely many patches in any tiling composed with the same prototiles. Figure 4 shows two small particular patches (darker colored tiles), called (long and short) bowties. A sequence of bowties placed side by side, as the colored tiles in Figure 5, is called a Conway worm. Any kites and darts tiling contains arbitrarily long finite worms, that cross each other.

A special marking of the tiles gives five sets of parallel straight lines, in five different directions, called Ammann bars (see [10 for more details). Figure 13 shows the marking, while Figure 5 shows one of these sets (red lines). The interval distance between any two consecutive bars in the same set can take only two values, such that the longer one, denoted by $L$, is $\varphi$ times the smaller

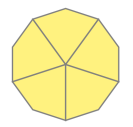

Sun

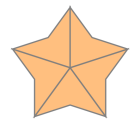

Star

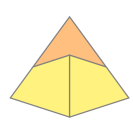

Ace

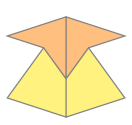

Deuce

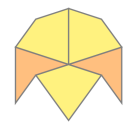

Jack

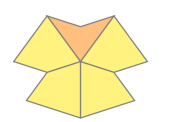

Queen

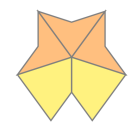

King

Fig. 3: The seven vertex configurations in a Penrose tiling by kites and darts. 


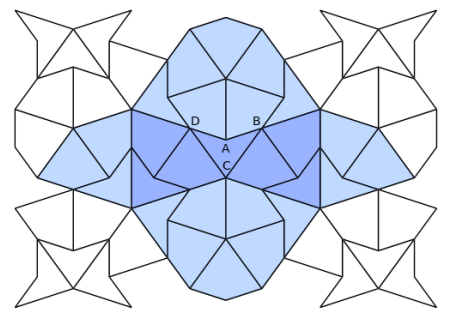

(a) Long bowtie in the queen's kingdom.

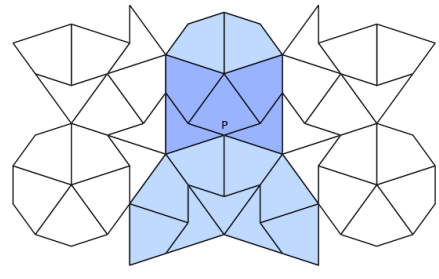

(b) Short bowtie in the jack's kingdom.

Fig. 4: Long and short bowties (darker colored tiles) respectively in the queen's and the jack's kingdoms (all colored tiles).

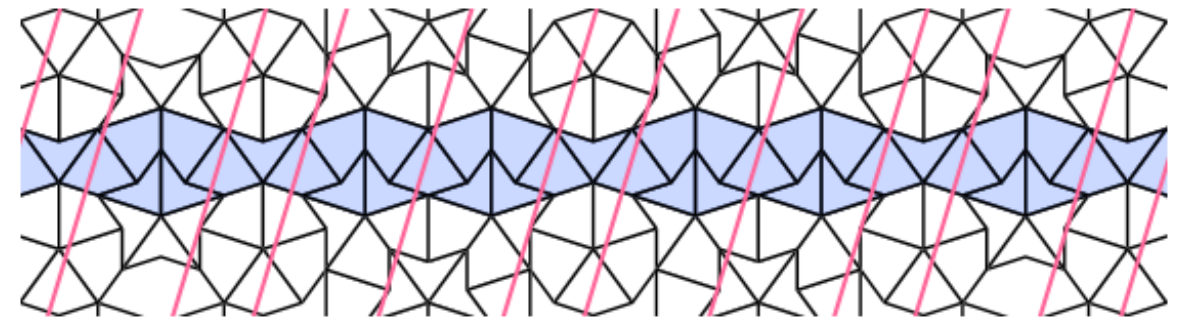

Fig. 5: A set of parallel Ammann bars along a Conway worm.

one, denoted by $S$. For a given set of Ammann bars, the sequence of $L$ 's and $S$ 's is called a musical sequence, and is a factor of the Fibonacci word. One significant property is that in a musical sequence we cannot have two $S$ 's in a row, nor three $L$ 's. Also, for a given length of the sequence, there are only two possible values for the number of $L$ intervals (see Proposition 4). This is why, starting with a given patch, Ammann bars often force the placement of some tiles outside the patch if one wishes to extend the tiling in order to cover the whole plane. In particular, each vertex configuration induces Ammann bars that force a (sometimes infinite) number of tiles in the plane and the set of all such tiles, along with the ones in the vertex configuration, is called an empire. We call kingdom the largest connected subset of an empire, which includes the vertex configuration. For instance, Figure 4 shows the queen's kingdom and the jack's kingdom.

\section{Early experiments}

Given a Penrose tiling, we are interested in the graph whose vertices are the tiles, and whose edges are given by the adjacency relation between tiles (see Figure 6). We call such a graph a Penrose graph and any of its induced subtree a Penrose tree. If the tree is a caterpillar, we simply call it a Penrose caterpillar. Due to the isomorphism property, graphs associated with kites and darts tilings all have the same leaf function, which we denote by $L_{k d}$. 


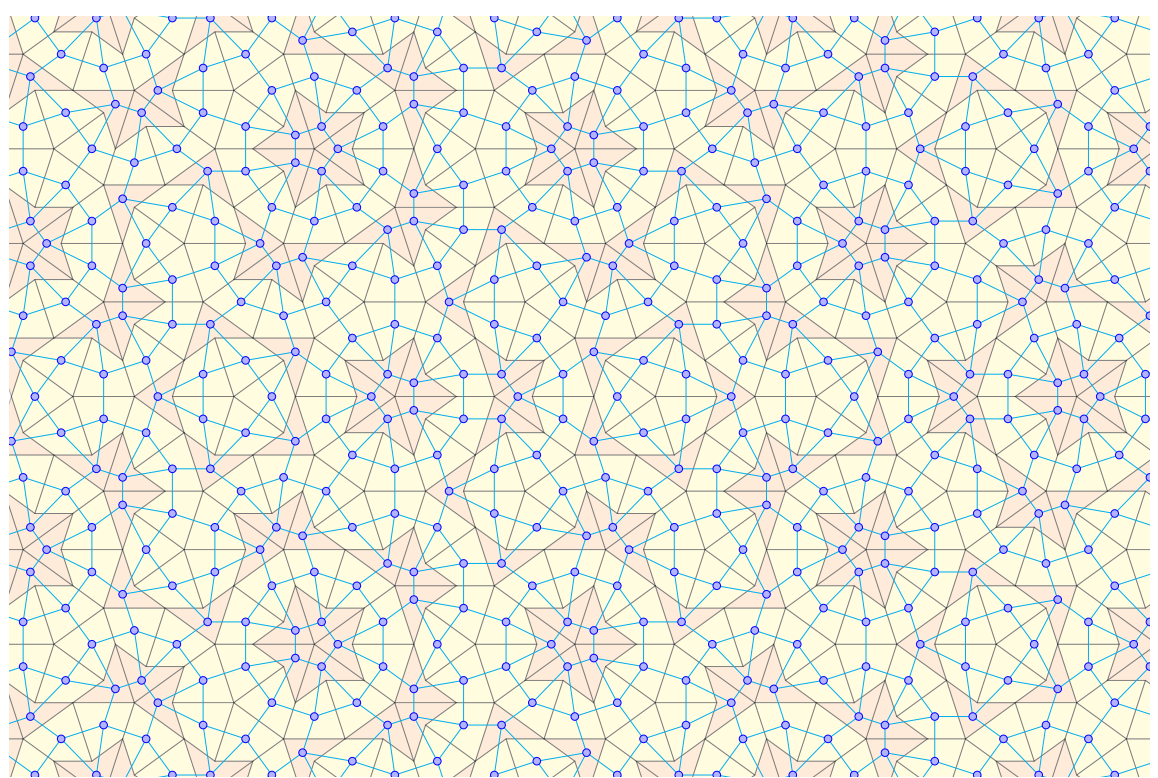

Fig. 6: A paler version of the Penrose tiling of Figure 1 superimposed with its underlying graph in blue.

There are a few ways to generate a Penrose tiling and several ways to encode the vertices of the tiling and of the graph. Though the projection method suggested by de Bruijn [6] is quite commonly used, we prefer the substitution method - subdividing the tiles like in Figure 2 and then scaling them - because it gives more insight on the structure and make it easier to build a discrete representation.

As a first step of computer exploration, we used the free and open-source software SageMath, since it facilitates graph manipulation and visualization. A tile is then simply defined by its type (kite or dart) and the coordinates of its corners expressed as complex numbers. Patches are generated from smaller patches using triangular decomposition, and the corresponding graph is defined by its list of tiles (vertices) and the adjacency function. After relabeling it, the generic algorithm for determining the leaf function of a finite graph given in [4 can be used. As an example, the Star vertex configuration was decomposed twice. The patch then obtained and its corresponding graph are shown in Figure 7. and the leaf function in table 1. The source code is available on Github.

A few problems occurred with these computations. First, due to the irrationality of the coordinates, we had approximation errors so we had to round values anytime a comparison was needed: the set structure did not eliminate doubles. Moreover, as the table suggests, even though there are 30 tiles in the patch, the values of the leaf function are correct up to $i=19$, but for $i \geq 22$ they do not correspond to those of the leaf function in the infinite graphs (which is nondecreasing, as shown in Section 4), and for $i=20,21$ we do not know whether they are correct. Trying to compute the leaf function of bigger patches becomes 


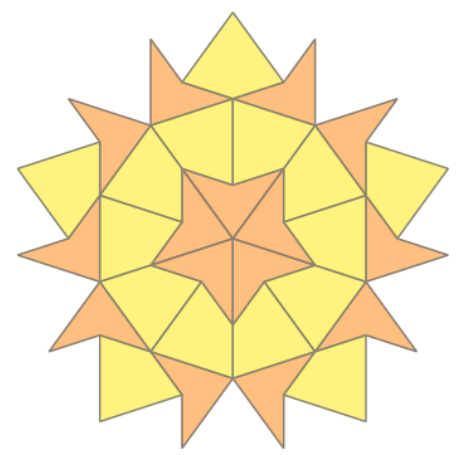

(a) Star after 2 decompositions

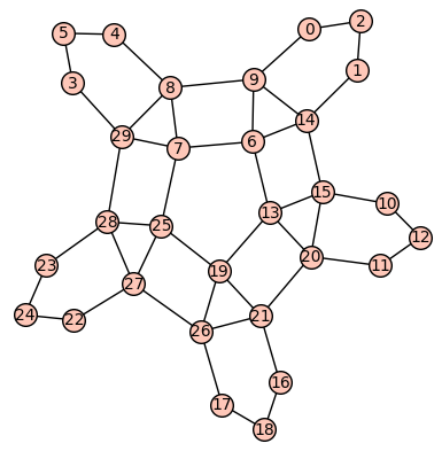

(b) Associated graph

Fig. 7: Star after 2 decompositions and its associated graph.

Table 1: Leaf function for the patch shown in Figure 7 (30 kites and darts).

$i \quad 001234456789101112131415161718192021222324252627282930$

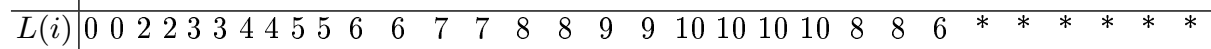

* denotes $-\infty$, when there are no induced subtrees of order $i$.

rapidly too long, since the time complexity of the generic, branch and bound algorithm is exponential. For instance, computations could not be completed overnight, while the program needs a few minutes for the patch in Figure 7.

These issues are the reasons why we soon focused on geometrical properties of the tilings, which allowed us to find the results of the next two sections. Since it remains difficult to even guess what the actual function is, because of nonperiodicity, new computational ideas have been developed in order to generate bigger patches faster and be able to see what induced subtrees look like.

\section{Upper bound for the leaf function of Penrose Trees}

Let us begin with an observation on $L_{k d}$ :

Proposition 1. The leaf function $L_{k d}$ of graphs corresponding to Penrose tilings is non-decreasing.

Proof. The proof naturally relies on the fact that we consider infinite graphs, but this condition is not enough. Let $n$ be an integer greater than 1 and $T$ a fully leafed induced subtree of order $n$. Recall that the vertices of $T$ are tiles (that is, subsets of the plane) and consider the convex hull $C$ of the union of these tiles. Then at worst all angles of $C$ have a measure of $4 \pi / 5$ and only a few configurations have to be examined considering a tile which lies in a corner. If the edge of the tile which lies on the boundary of $C$ is a long one, then adding a tile adjacent to it that is not in $C$ will not create a cycle (due to the angles). Otherwise we have a kite with its short side on the boundary of $C$, so we can add another kite exterior to $C$ (like in the deuce configuration, without the darts) 
without creating a cycle. In both cases we obtain a tree of order $n+1$ with at least as many leaves as $T$, so $L_{k d}(n+1) \geq L_{k d}(n)$.

Since any tile in a kites and darts tiling is adjacent to four other tiles, the maximum degree of a vertex in a Penrose tree cannot be more than 4 . We actually have:

Proposition 2. The maximal degree of a vertex in a Penrose tree is 3.

Proof. For any kite or dart in a Penrose tree $T$, at most 3 of its adjacent tiles can also be in $T$. Firstly, any dart lies in an ace for it is the only possible vertex configuration for the vertex in the reflex angle of the dart. It follows that if the dart is in $T$, then only one among two (adjacent) kites in the corresponding ace can be in $T$. As for the kites, two cases are to be considered, shown in Figure 8. If we focus on the side $C D$ of the red kite $8 \mathrm{a}$, there is exactly one way to arrange a dart adjacent to it (Case 1) and exactly one way to arrange a kite adjacent to it (Case 2). In Case 1, the yellow kite is forced so that if $A B C D$ is in $T$ then the dart and the yellow kite cannot be both in $T$. In Case 2, vertex $C$ can only be in a deuce, so that the darts are forced and then so is the kite adjacent to $A B$. Subsequently, if the red kite is in $T$ then $T$ cannot contain both the tiles adjacent to $A B$ and $B C$.

This constraint on degrees turns out to be useful in finding an upper bound for $L_{k d}$, and more generally for the leaf function in any graph sharing the same constraint on degrees. To prove proposition 3 , we need the following lemma:

Lemma 1. Let $L$ be the leaf function of a graph $G$ such that the maximal degree of a vertex in any induced subtree of $G$ is at most 3. Then for any positive integer $n$, if $L(n)>L(n-1)$ we have $L(n+1) \leq L(n)$.

Proof. Let $n$ a positive integer such that $L(n)>L(n-1)$. Suppose $L(n)<$ $L(n+1)$ and let $T_{n+1}$ be a fully leafed induced subtree of $G$ of order $n+1$. Since $L(n)<L(n+1)$, by deleting a leaf $f$ from $T_{n+1}$ we get a tree $T_{n}$ whose number of leaves $\left|T_{n}\right|$ is greater or equal to $L(n)$, so $\left|T_{n}\right|$ 因 $=L(n)$ by maximality of the leaf function. Let $v$ be the vertex of $T_{n}$ adjacent to $f$ in $T_{n+1}$, then $v$ cannot be a leaf in $T_{n}$ because $\left|T_{n}\right|$ 因 $=L(n)<L(n+1)=\left|T_{n+1}\right|$. Suppose $v$ has degree 0 , then $T_{n}$ is of order 1 so $\left|T_{n}\right| \otimes=L(1)=L(0)$, which contradicts the

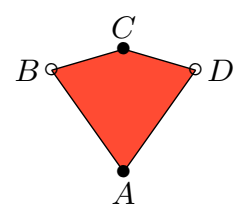

(a) Kite

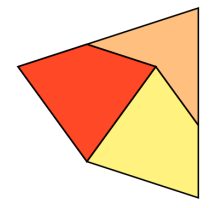

(b) Case 1

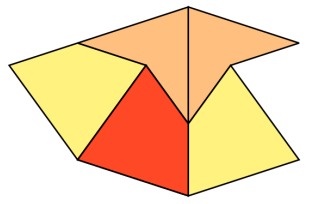

(c) Case 2

Fig. 8: Adjacency constraints on kites in Penrose trees: for a kite $A B C D$ (red), when focusing on the side $C D$, only two cases are possible. 
assumption $L(n)>L(n-1)$. Hence $v$ has degree at least 2 in $T_{n}$. Without loss of generality, we can choose $f$ so that $v$ is adjacent to at most one inner vertex of $T_{n+1}$. If $v$ had degree 3 in $T_{n}$ then it would have degree 4 in $T_{n+1}$, which is impossible. Finally if $v$ has degree 2 in $T_{n}$, since at most one of its neighbors is an inner one, there is a leaf $f^{\prime}$ adjacent to $v$ in $T_{n}$. Let $T_{n-1}$ be the tree obtained by deleting $f^{\prime}$ from $T_{n}$, then $v$ is a leaf in $T_{n-1}$ so we have $\left|T_{n-1}\right|_{\text {因 }}=\left|T_{n}\right|_{\text {因 }}=L(n)>L(n-1) \geq\left|T_{n-1}\right|$ 因, which is absurd.

Proposition 3. Let $L$ be the leaf function of a graph $G$ such that the maximal degree of a vertex in any induced subtree of $G$ is at most 3. Then for any nonnegative integer $n, L(n) \leq\lfloor n / 2\rfloor+1$.

Proof. In any graph, the inequality is true for $n \in[0,1,2]$. Lemma 1 then gives the inductive step, provided that induced subtrees of orders $n$ and $n+1$ exist: if $T_{n+1}$ is a fully leafed Penrose tree of order $n+1$, then by removing a leaf from $T_{n+1}$ we obtain a tree $T_{n}$ of order $n$ which has at least $\left|T_{n+1}\right|$ 四 -1 leaves, so that $L(n) \geq L(n+1)-1$. The difference between $L(n)$ and $L(n+1)$ is at most 1 and these values are equal for at least half the values of $n$, hence the result for all $n \in[0 . . k]$, where $k$ is the greatest integer for which an induced subtree of order $k$ exists. Finally, for all $n>k$, since there is no subtree of order $n$ we have $L(n)=-\infty \leq\lfloor n / 2\rfloor+1$.

With Propositions 2 and 3 , we have proved:

Theorem 1. For any non-negative integer n,

$$
L_{k d}(n) \leq\lfloor n / 2\rfloor+1 .
$$

Thanks to the algorithm presented in [4] and a particular Penrose tree (see Figure 9), we know that this upper bound is reached for all $n \leq 19$ (except for $n=1$ ) and we thinj that it is not realized for $n \geq 20$ but we cannot prove it yet.

\section{Penrose caterpillars and lower bound}

As mentioned in Section 2.2 arbitrarily long Conway worms cross Penrose tilings, and by local isomorphism there are infinitely many of them. Figure 5 shows how they correspond to parallel Ammann bars, so that we can use the properties of musical sequences: any subtree whose vertices are all tiles inside the worm is necessarily a path. It is then easy to build caterpillars whose derived paths are inside the worms.

This section gives an increasing family of Penrose trees with the maximum number of leaves among such caterpillars. In the best case scenario, we will have fully leafed Penrose trees; in the worst case scenario, we will have a lower bound for the leaf function, thus complementing the upper bound given in Theorem 1 . The idea of caterpillars is suggested by the fact that fully leafed induced subtrees in the triangular and hexagonal lattices are caterpillars, or almost caterpillars, and the maximum degree of a vertex in a Penrose tree is 3 as in these lattices. 


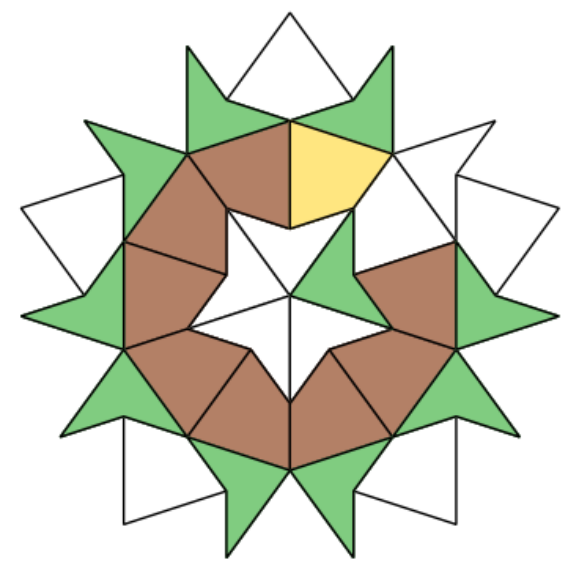

Fig. 9: A fully leafed Penrose tree with 19 vertices and 10 leaves. Note that this is a caterpillar. Tiles are colored with respect to their degrees.

Recall Figure 4 which shows the queen's kingdom around vertex $C$ and the jack's kingdom around vertex $P$ (colored tiles). We use the observations on kingdoms and bowties to show how tiles adjacent to a worm are forced.

Lemma 2. Any long bowtie lies in a queen's kingdom.

Proof. In Figure 4 a notice that the dart in the center of the long bowtie forces two kites above it, so that the vertex configuration around point $A$ is an ace. Moreover, since this dart is surrounded with two aces in the bowtie, the vertex configurations at points $B$ and $D$ are necessarily jacks, forcing the vertex neighborhood around $C$ to be a queen (using the jack's kingdom). Hence the long bowtie is entirely inside a queen's kingdom.

Lemma 3. Any short bowtie lies in a jack's kingdom.

Proof. In Figure $4 \mathrm{~b}$ the vertex neighborhood around $P$ can only be a jack, then we can see how the short bowtie is inside the jack's kingdom.

Now let us first consider the patch composed with a short bowtie and its adjacent tiles. Figure 10a shows a fully leafed Penrose caterpillar $C_{S}$ in such a patch: this caterpillar has 7 vertices including 4 leaves, and we know that $L_{k d}(7) \leq 4$ so it is a fully leafed Penrose tree in the whole tiling. In this patch, this is the biggest fully leafed Penrose tree and it is unique up to isometry. Furthermore, it is easily extendable on both sides (left and right) so that the derived path of $C_{S}$ stays in the worm. In the patch composed with the long bowtie and its adjacent tiles, as shown in Figure $10 \mathrm{~b}$ the biggest fully leafed Penrose tree that can be extended on both sides following the worm has 11 vertices and 6 leaves. It is also unique up to isometry and it is a caterpillar which we call $C_{L}$. 


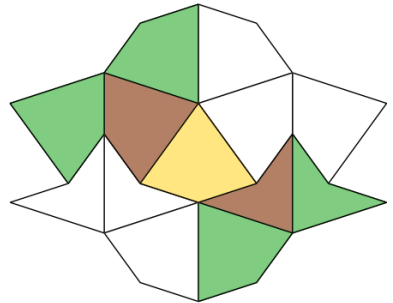

(a) $C_{S}$

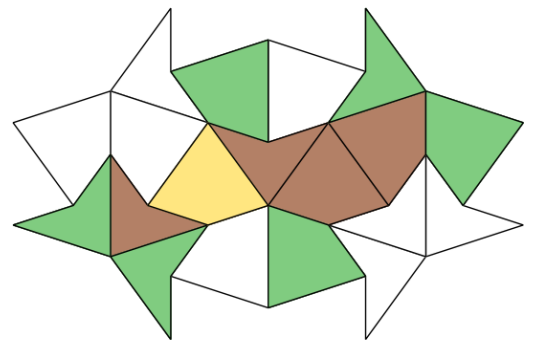

(b) $C_{L}$

Fig. 10: Fully leafed Penrose caterpillars in bowtie patches: (a) (respectively (b)) Fully leafed caterpillar in the patch composed with the short (resp. long) bowtie and its adjacent tiles. Tiles are colored with respect to their degrees.

With both patches side by side it is easy to see what happens when a short and a long bowtie follow each other in a worm: if for instance the short one is on the left, both darts in $C_{S}$ coincide with the two ones on the left side of $C_{L}$, so that we lose two leaves but the resulting subgraph is a tree. Then we have a caterpillar of order 16 with $4+6-2=8$ leaves, and the same happens when the short bowtie is placed to the right of the long one (except the tiles that coincide are kites). Note that this caterpillar is not fully leafed, so the function to be presented here is not the leaf function but only a lower bound for it. Since a worm cannot have two consecutive short bowties, the only remaining possible configuration is two long bowties in a row. In this case, one caterpillar is the reflection of the one in Figure $10 \mathrm{~b}$ and the concatenation, in either way, gives a caterpillar with $2 \cdot 6-2=10$ leaves and 20 vertices. Figure 11 gives an example of a Penrose caterpillar constructed along a Conway worm.

Let $C_{S}^{\prime}$ and $C_{L}^{\prime}$ be the caterpillars obtained from $C_{S}$ and $C_{L}$ by deleting the leaves on the sides - the ones that eventually coincide with inner vertices during concatenation -, then $C_{S}^{\prime}$ has 2 leaves and 5 vertices, and $C_{L}^{\prime}$ has 4 leaves and 9 vertices. Let $q_{S}$ (resp. $q_{L}$ ) be the ratio of number of leaves over order in $C_{S}^{\prime}$ (resp. $C_{L}^{\prime}$ ), then $q_{S}=2 / 5$ is lower than $q_{L}=4 / 9$. So we consider caterpillars that are concatenations of $C_{S}^{\prime}$ and $C_{L}^{\prime}$, and we would like them to lie on as many

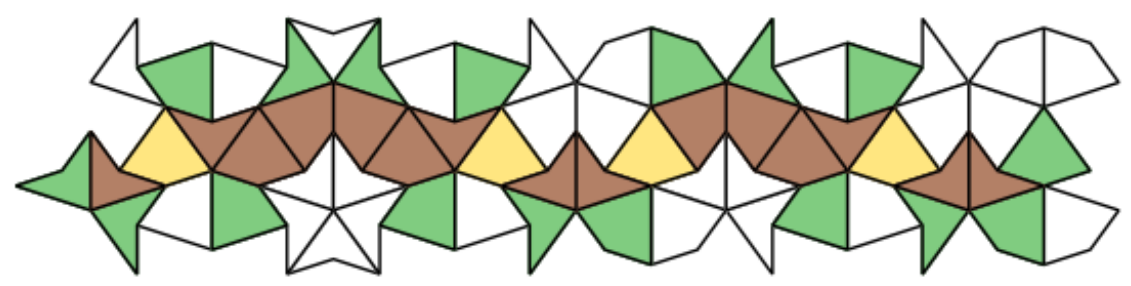

Fig. 11: A Penrose caterpillar along a worm, with 17 leaves and 36 vertices. Tiles are colored with respect to their degrees. 
long bowties as possible. Figure 5 shows how a set of parallel Ammann bars determines a sequence of bowties in a worm: each bowtie (long or short) crosses two half $L$-intervals, and each long bowtie additionally crosses one $S$-interval between both $L$ 's. Thus each long bowtie corresponds to $L+S$ and each short bowtie to $L$. Hence $L$ corresponds to $C_{S}^{\prime}$ which means 2 leaves in 5 tiles, and $S$ corresponds to what is left of $C_{L}^{\prime}$ when tiles corresponding to $L$ are removed, that is 2 leaves in 4 tiles.

As a result, we can now focus only on $L$ and $S$ intervals. Since each interval give 2 leaves, we just have to determine the number $k$ of intervals in the caterpillar and how many of them are long. Before completing our main argument, we need a last proposition.

Proposition 4 (Proposition 10.6.10 of [10]). If $k$ is the number of intervals the caterpillar crosses, then the number $x_{k}$ of $L$ intervals can only take two values: $x$ and $x+1$ such that

$$
\frac{x}{k}<\frac{1}{\varphi}<\frac{x+1}{k} .
$$

Hence, we have $x_{k}=\lfloor k / \varphi\rfloor$ or $x_{k}=\lceil k / \varphi\rceil$. The correct value will actually be the one that best approximates $1 / \varphi$, and when we need to choose, since we would not want to exceed the real value for $L_{k d}(n)$, we take the value that corresponds to less $L$ intervals, that is $x_{k}=\lfloor k / \varphi\rfloor$.

If $n$ is the order of the caterpillar and $k$ is the number of intervals in it, since we prefer to take a smaller value for $k$ and then add a few tiles to equal $n$, then we want the greatest $k$ such that

$$
5 \frac{k}{\varphi}+4\left(k-\frac{k}{\varphi}\right) \leq n
$$

that is

$$
\frac{k}{\varphi}+4 k \leq n
$$

then

$$
k\left(\frac{4 \varphi+1}{\varphi}\right) \leq n
$$

which yields

$$
k \leq \frac{\varphi n}{4 \varphi+1} .
$$

So finally we have

$$
k=\left\lfloor\frac{\varphi n}{4 \varphi+1}\right\rfloor .
$$

The number of leaves over the intervals is then $2 k$ and since $4<(4 \varphi+1) / \varphi<$ 5 there are at most 5 remaining tiles to arrange at one end of the caterpillar or the other. Let $\Delta$ be the number of remaining tiles. If $0 \leq \Delta \leq 2$ then we just have to add $\Delta$ leaves to the ends ; if $3 \leq \Delta \leq 4$ the best strategy is to add one 
tile (a leaf) to an end and 2 or 3 tiles on the other, giving respectively 1 or 2 leaves; if $\Delta=5$ then we add 3 tiles $/ 2$ leaves to an end and 2 tiles/ 1 leaf to the other. We have proved:

Theorem 2. Let $\ell_{k d}(n)$ denote the number of leaves of a caterpillar of order $n$ constructed as described above. Then $\ell_{k d}$ is a lower bound for the leaf function $L_{k d}$ and we have

$$
\ell_{k d}(n)=2 k+ \begin{cases}\Delta & \text { if } 0 \leq \Delta \leq 2 \\ \Delta-1 & \text { if } 3 \leq \Delta \leq 4 \\ 3 & \text { if } \Delta=5\end{cases}
$$

where $k=\lfloor\varphi n /(4 \varphi+1)\rfloor$ and $\Delta=n-4 k-\lfloor k / \varphi\rfloor+1$.

For instance, the caterpillar in Figure 11 has $n=36$ vertices and 17 leaves, and we can verify that $k=7$ and $\Delta=5$, which yields $\ell_{k d}(36)=17$. By removing the 5 rightmost tiles, we would get a caterpillar with 31 vertices and 15 leaves, and we have $\ell_{k d}(31)=15$. Finally, from Theorems 1 and 2 we have

Corollary 1. For all $n \in \mathbb{N}$,

$$
2 \varphi n /(4 \varphi+1) \leq L_{k d}(n) \leq\lfloor n / 2\rfloor+1 .
$$

Since $2 \varphi /(4 \varphi+1) \simeq 0.433$, the gap between lower and upper bounds for the leaf function $L_{k d}$ is relatively small. Yet it remains difficult to even guess what the actual leaf function is, especially if we do not know what the fully leafed Penrose trees look like. Hence we would like to efficiently be able to generate at least some of them.

\section{Discrete representation for computing Penrose tilings}

In the square and cubic lattices, the coordinates of the vertices are simply the tuples of integers (respectively in $2 \mathrm{D}$ and $3 \mathrm{D}$ ) ; in the triangular and hexagonal lattices, the graphs are isomorphic to ones with only regular integer coordinates. In the case of Penrose tilings, identifying points with respect to a coordinate system is more intricate.

Let us call a Penrose point any point of $\mathbb{R}^{2}$ that is the vertex of at least one Penrose tile and let $\mathcal{P} \subseteq \mathbb{R}^{2}$ be the set of all Penrose points. Assuming that the plane origin is the vertex of at least one tile, it is easy to see that, for any $p \in \mathcal{P}$, there exist 20 integers $a_{k}$ and $b_{k}$, for $k=0,1, \ldots, 9$, such that

$$
p=\sum_{k=0}^{9}\left(a_{k}+b_{k} \varphi\right) e^{k i \pi / 5} .
$$

Indeed, when moving along the edges of the tiles, there are 10 possible directions, given by $e^{k i \pi / 5}$ for $k=0,1, \ldots, 9$, and 2 possible steps, either by a unit or a $\varphi$ distance. Hence, from a computational perspective, the location of any vertex of a tile can be represented by 20 integers. This representation is not unique, which 
makes it harder to decide whether two Penrose points are the same. Fortunately, a unique representation can be derived, as we see in the following paragraphs.

For any integer $\ell$, let

$$
B(\ell)=\left[e^{(\ell+k) i \pi / 5}\right]_{k=1,2,3,4}
$$

We first prove that any Penrose point can be uniquely represented with respect to $B(\ell)$.

Theorem 3. For any integer $\ell$ and Penrose point $p$, there exist four unique integers $a_{0}, a_{1}, a_{2}$ and $a_{3}$ such that

$$
p=\sum_{k=1}^{4} a_{k} b_{k}
$$

where $B(\ell)=\left[b_{k}\right]_{k=1,2,3,4}$

Proof. Without loss of generality, we only prove the case $\ell=-1$.

(Existence) Using Equation 1, we can write

$$
p=\sum_{k=0}^{9}\left(a_{k}^{\prime}+b_{k}^{\prime} \varphi\right) e^{k i \pi / 5}
$$

for some integers $a_{k}^{\prime}, b_{k}^{\prime}$, with $k=0,1, \ldots, 9$. We claim that the integers $a_{0}, a_{1}$, $a_{2}$ and $a_{3}$ are given as follows:

$$
\begin{aligned}
& a_{0}=\left(a_{0}^{\prime}+b_{9}^{\prime}+b_{1}^{\prime}\right)-\left(a_{5}^{\prime}+b_{4}^{\prime}+b_{6}^{\prime}\right)-a_{4}, \\
& a_{1}=\left(a_{1}^{\prime}+b_{0}^{\prime}+b_{2}^{\prime}\right)-\left(a_{6}^{\prime}+b_{5}^{\prime}+b_{7}^{\prime}\right)+a_{4}, \\
& a_{2}=\left(a_{2}^{\prime}+b_{1}^{\prime}+b_{3}^{\prime}\right)-\left(a_{7}^{\prime}+b_{6}^{\prime}+b_{8}^{\prime}\right)-a_{4}, \\
& a_{3}=\left(a_{3}^{\prime}+b_{2}^{\prime}+b_{4}^{\prime}\right)-\left(a_{8}^{\prime}+b_{7}^{\prime}+b_{9}^{\prime}\right)+a_{4}
\end{aligned}
$$

where $a_{4}=\left(a_{4}^{\prime}+b_{3}^{\prime}+b_{5}^{\prime}\right)-\left(a_{9}^{\prime}+b_{8}^{\prime}+b_{0}^{\prime}\right)$.

In order to substantiate our claim, we first provide three identities that hold for any integer $k \in\{0,1, \ldots, 9\}$, where the addition and substraction is taken modulo 10:

(1) $e^{k i \pi / 5}=-e^{(k+5) i \pi / 5}$, obtained by applying a rotation of angle $\pi$;

(2) $\varphi e^{k i \pi / 5}=e^{(k+1) i \pi / 5}+e^{(k-1) i \pi / 5}$, since $\varphi=2 \cos (\pi / 5)$ and

(3) $\sum_{k=0}^{4}(-1)^{k+1} e^{k i \pi / 5}=0$, using the relation $\varphi^{2}=1+\varphi$. 
Therefore,

$$
\begin{aligned}
\sum_{k=0}^{3} a_{k} e^{k i \pi / 5}= & \left(\sum_{k=0}^{4} a_{k} e^{k i \pi / 5}\right)-a_{4} e^{4 i \pi / 5} \\
= & \sum_{k=0}^{4}\left(a_{k}^{\prime}-a_{k+5}^{\prime}\right) e^{k i \pi / 5}+\sum_{k=0}^{4}\left(b_{k}^{\prime} e^{(k+1) i \pi / 5}-b_{k}^{\prime} e^{(k+4) i \pi / 5}\right) \\
& +\sum_{k=5}^{9}\left(b_{k}^{\prime} e^{(k-1) i \pi / 5}-b_{k}^{\prime} e^{(k-4) i \pi / 5}\right)+\sum_{k=0}^{4}(-1)^{k+1} a_{4} e^{k i \pi / 5} \\
= & \sum_{k=0}^{4} a_{k}^{\prime} e^{k i \pi / 5}+\sum_{k=5}^{9} a_{k}^{\prime} e^{k i \pi / 5}+\sum_{k=0}^{4}\left(b_{k}^{\prime} e^{(k+1) i \pi / 5}+b_{k}^{\prime} e^{(k-1) i \pi / 5}\right) \\
& +\sum_{k=5}^{9}\left(b_{k}^{\prime} e^{(k-1) i \pi / 5}+b_{k}^{\prime} e^{(k+1) i \pi / 5}\right)+0 \\
= & \sum_{k=0}^{9}\left(a_{k}^{\prime}+b_{k}^{\prime} \varphi\right) e^{k i \pi / 5}
\end{aligned}
$$

as claimed. Note that the penultimate equality follows from Identities (1) and (3), and the last equality from Identity (2).

(Unicity) It is sufficient to prove that the set

$$
\left\{e^{k i \pi / 5} \mid k=0,1,2,3\right\}
$$

is linearly independent over $\mathbb{Z}$, that is,

$$
\sum_{k=0}^{3} a_{k} e^{k i \pi / 5}=0 \text { implies } a_{k}=0 \text { for } k=0,1,2,3 .
$$

Since $\cos (3 \pi / 5)=-\cos (2 \pi / 5)$ and $\sin (3 \pi / 5)=\sin (2 \pi / 5)$, one first shows that

$$
\begin{aligned}
0 & =\sum_{k=0}^{3} a_{k} e^{k i \pi / 5} \\
& =a_{0}+a_{1} e^{i \pi / 5}+\left(a_{2}-a_{3}\right) \cos (2 \pi / 5)+i\left(a_{2}+a_{3}\right) \sin (2 \pi / 5) .
\end{aligned}
$$

Next, using the double angle identities for both sin and cos, and after factoring and regrouping, we find

$$
\begin{aligned}
0= & a_{0}+a_{2}-a_{3}+\cos (\pi / 5)\left(a_{1}+2\left(a_{2}-a_{3}\right) \cos (\pi / 5)\right) \\
& +i \sin (\pi / 5)\left(a_{1}+2\left(a_{2}+a_{3}\right) \cos (\pi / 5)\right) .
\end{aligned}
$$

But all $a_{k}$ are integers, and we know that $\cos (\pi / 5), \sin (\pi / 5)$ are not, which implies $a_{2}=a_{3}, a_{1}=0, a_{0}=0$ and $a_{2}=-a_{3}$, i.e. $a_{0}=a_{1}=a_{2}=a_{3}=0$, concluding the proof. 


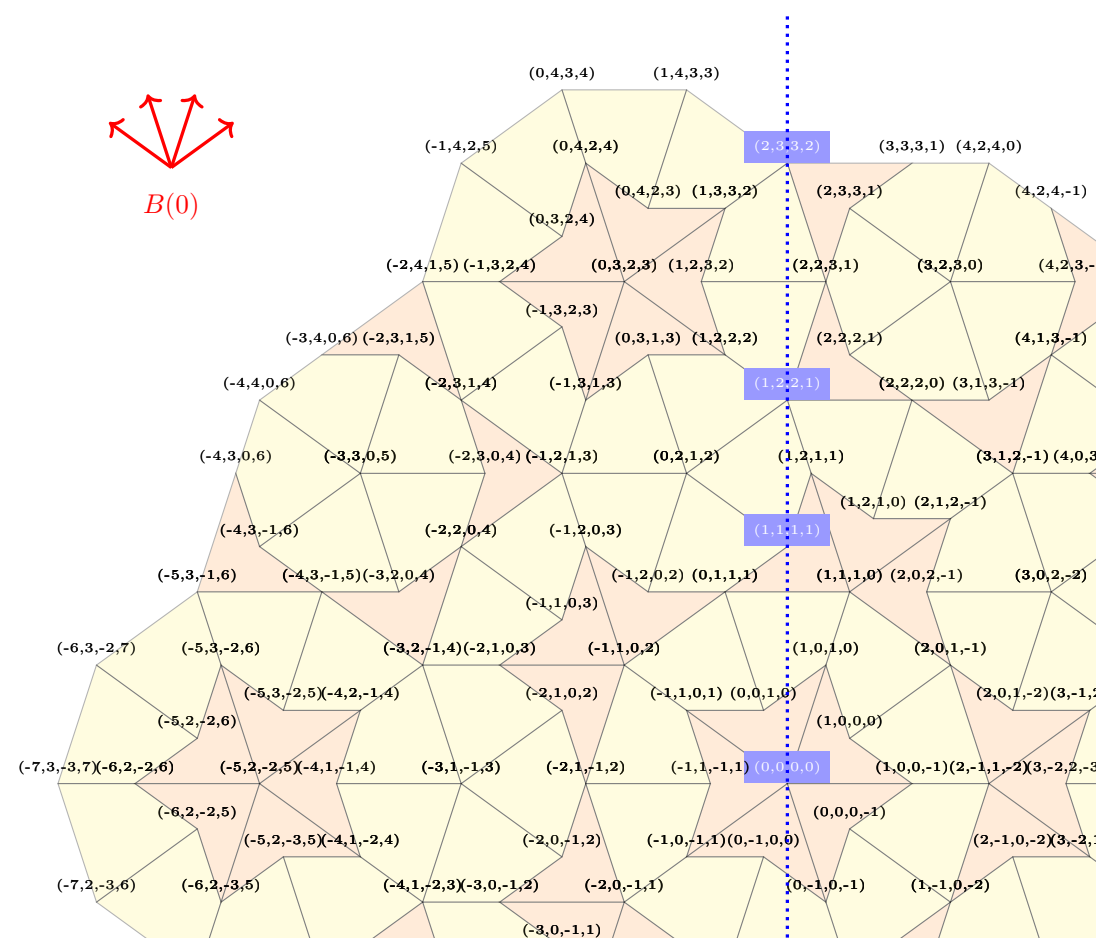

(a) Along the angle $0+\pi / 2$

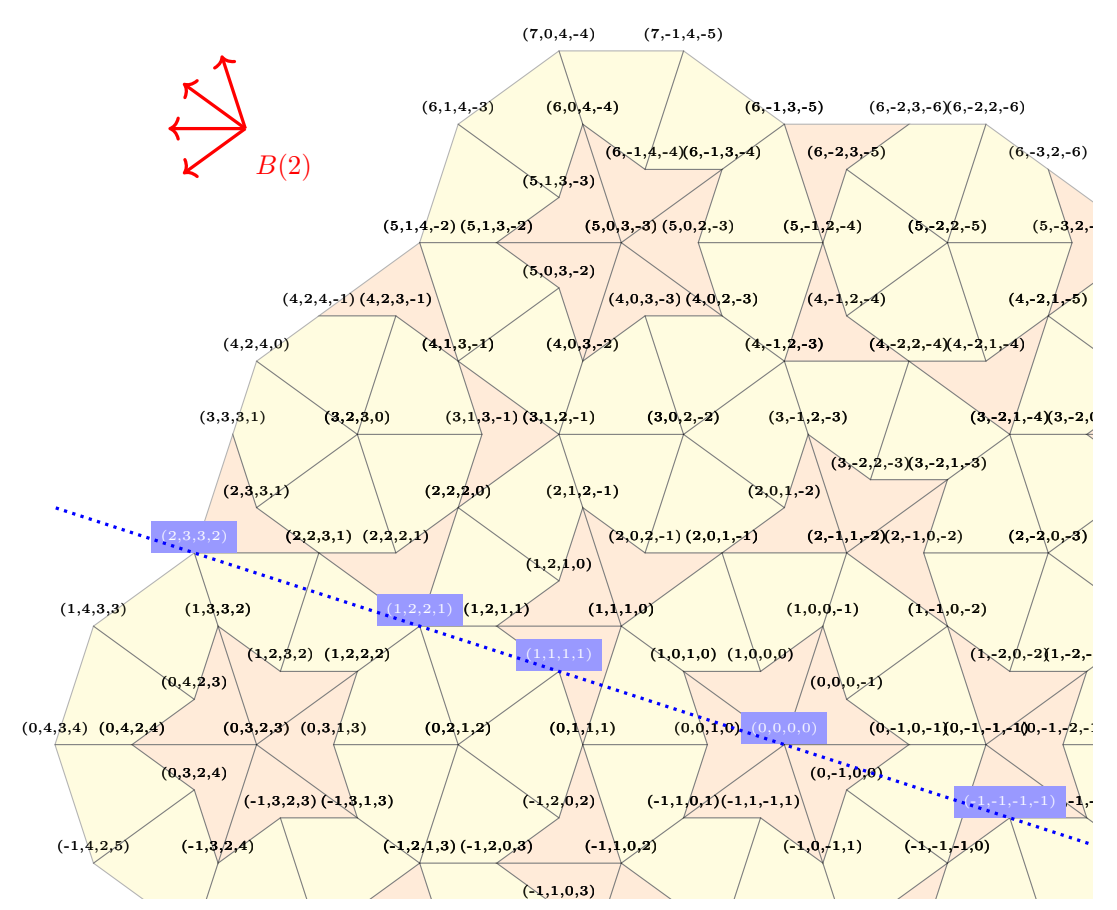

(b) Along the angle $2 \pi / 5+\pi / 2$

Fig. 12: The coordinates of the points taken in the same patch, but with respect to two different bases. (a) The basis $B(0)=\left\{e^{k i \pi / 5} \mid k=1,2,3,4\right\}$. (b) The basis $B(2)=\left\{e^{k i \pi / 5} \mid k=3,4,5,6\right\}$. 
For any integer $\ell$, let $f_{B(\ell)}: \mathbb{Z}^{20} \rightarrow \mathbb{Z}^{4}$ be the function that maps any 20tuple integers onto $\left(a_{0}, a_{1}, a_{2}, a_{3}\right)$, where $a_{0}, a_{1}, a_{2}$ and $a_{3}$ are defined in the proof of Theorem 3 . Let $p$ and $p^{\prime}$ be two Penrose points and $f(p), f\left(p^{\prime}\right)$ be the unique 20-tuple obtained by applying $f$ to any 20-tuple representation of $p$ and $p^{\prime}$ respectively. Then the following statement hold:

1. We have $p=p^{\prime}$ if and only if $f_{B(\ell)}(p)=f_{B(\ell)}\left(p^{\prime}\right)$.

2. The relation defined by $p \preceq_{B(\ell)} p^{\prime}$ if and only if $f_{B(\ell)}(p) \leq f_{B(\ell)}\left(p^{\prime}\right)$, where $\leq$ denotes the lexicographical order, is a total order relation.

3. Two Penrose tiles are equal if and only if their origins are equal and their orientations are the same.

4. Two Penrose tiles are adjacent if and only if the share exactly two points.

Moreover, except for the drawing parts, all computations on Penrose tilings and Penrose graphs can be performed exclusively on integers and are therefore not sensible to floating numerical errors. The reader is invited to look at [16], where similar ideas were used for representing Penrose points by four integers, although the representation is different and is for tilings by Penrose rhombs.

\section{Dynamic generation of induced subtrees}

Since enumerating all possible induced subtrees of size $n$ takes too long as $n$ gets large, we would like to generate fully leafed Penrose trees, by trying to have as many inner vertices of degree 3 as possible. But generating induced subtrees can be tricky: as mentioned before, if we build a tree with kites and darts using only local rules there is no guarantee that our tree is actually a Penrose tree. Since we would like to be able to expand the tree in any direction, we can exploit the constraints induced by the Ammann bars. From now on, we shall call the sets of all parallel Ammann bars Ammann sets. Note that the angle between two of them is a multiple of $\pi / 5$.
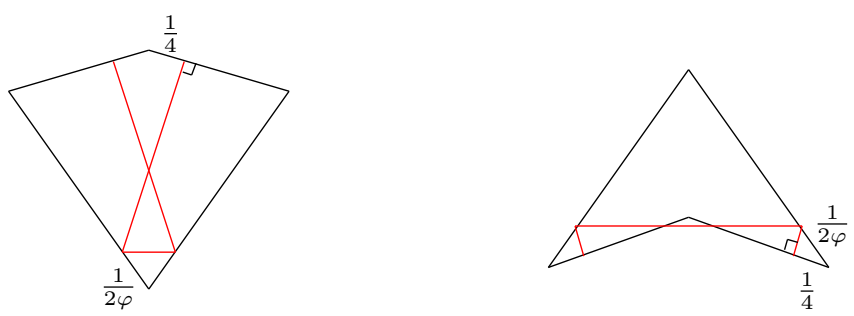

Fig. 13: Ammann segments on kites and darts. The lengths of the sides are 1 and $\varphi$, segments respect the symmetry of the tiles. Note that the conditions on Ammann bars prevents not respecting the assembly rules of the tiling: endpoints of the segments on adjacent tiles have to coincide. 


\subsection{Constraints on Ammann sets}

Usually, a line $\Delta$ can be represented in a computer by a pair $(p, \theta)$, where $p$ is a point through which $\Delta$ goes and $\theta$ is a direction. In the special case of Ammann bars, the direction can be represented by taking the angle of the normal vector of the bar (which is a multiple of $\pi / 5$ ), but it is easy to see that the bars never cross any Penrose point (see Figure 13).

Fortunately, by carefully choosing a Penrose point whose distance with the Ammann bar is constant, we can build a completely discrete representation. Any representative could be used, but for sake of visualization, we choose a point in the concave part of a dart, since the Ammann bar passes close to it. Therefore, the distance between the representative and the line is $\approx 0.059$. In order to make sure that a dynamically constructed tree of tiles is valid, we need to maintain the sets of parallel Ammann bars as described in Invariant 1.

\section{Invariant 1:}

- Each Ammann set $A$ has angle $k \pi / 5$, where $k \in\{0,1,2,3,4\}$.

- In a given set, each bar $B$ is represented by a Penrose point $p$ whose distance with respect to $B$ is $\approx 0.059$ in the $k \pi / 5+\pi$ direction. Notice that the exact value of the distance is not relevant, since it is not needed in the computations. In other words, we represent Ammann sets by translated copies, which does not cause any problem since we are interested in the distance between consecutive bars.

- These representatives remain sorted in increasing order (see Theorem 4 further) along the direction, as new bars are added to the set.

- The distance between two consecutive bars can only take two values: $S$ and $L$. More precisely, the sequence of these distances is a binary word over $\{L, S\}$ which must be a musical sequence.

\subsection{Procedure to generate valid patches}

Given the previous invariant, we can derive a procedure to build valid patches as follows. We start with a patch composed of a single tile, either a dart or a kite, located at the origin. At each step, we try to add a new tile $t$ that is a neighbor of any tile in the current patch. For the new tile to be allowed, there are a few conditions to examine (see Figure 14):

1. Check if it yields a valid vertex configuration: some partial configurations can only be completed in one way. If this step is neglected, some problem can arise (see Figure 14(c)).

2. Check if all Ammann segments in $t$ correspond to Ammann bars that are already in our sets: if so, then adding $t$ is correct (see Figure 14(b));

3. For each of the 3 Ammann segments in $t$, temporarily update the current Ammann sets accordingly:

(a) Let $d, d^{\prime}$ denote the distances between $B$ and the two bars in the set which are closest to $B$, then $d$ and $d^{\prime}$ should be in $\{S, L, S+L, 2 L\}$. 


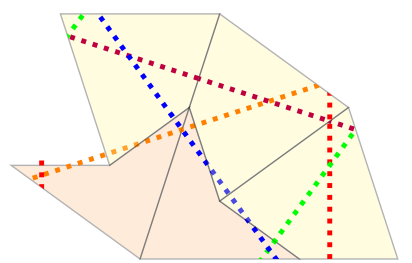

(a) Valid patch

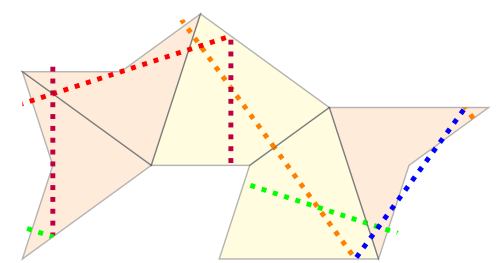

(b) Alignment problem

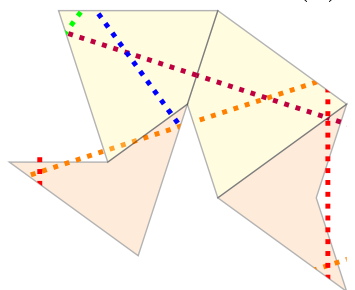

(c) Vertex configuration problem

Fig. 14: Examples of valid and non valid patches. (a) The Ammann bars are compatible. (b) The green Amman segments are not on the same bar, and the distance between them is too small. (c) The two darts are not compatible: if the leftmost one stays, then we must complete the thin hole with another dart, like in Subfigure (a).

(b) If the previous condition is met, then check whether the new word thus obtained is a musical sequence.

As soon as (a) or (b) is not correct, then $t$ should not be added so we backtrack to the previous state of each Ammann set that was temporarily changed for $t$.

Algorithm 1 details how to check whether a binary word $w$ over $\{L, S\}$ is a musical sequence or not. One can summarize it as follows: if $w$ contains at most one letter then it is a musical sequence, else if the number $x_{k}$ of occurrences of $L$ in $w$ is not as stated in Proposition 4 or if $w$ contains " $S S$ " or " $L L L$ " then it is not a musical sequence, else a new (shorter) word $w^{\prime}$ is obtained by composing $w$ according to the following substitution rules:

$$
S \rightarrow L, \quad L L \rightarrow S, \quad L \rightarrow \lambda
$$

where $\lambda$ denotes the empty string, and we recursively check whether $w^{\prime}$ is a musical sequence. Note that local rules of the Fibonacci word can be represented by an automaton but as in the case of Penrose tilings, they are not sufficient to ensure that a binary word is indeed a musical sequence. The above procedure was described summarily in [9, but for sake of thoroughness, we include the formal algorithm here.

\subsection{Last computational considerations}

As a new tile is added while maintaining connectivity, at most two new Ammann bars are introduced. For each of them, a representative can be chosen and the 


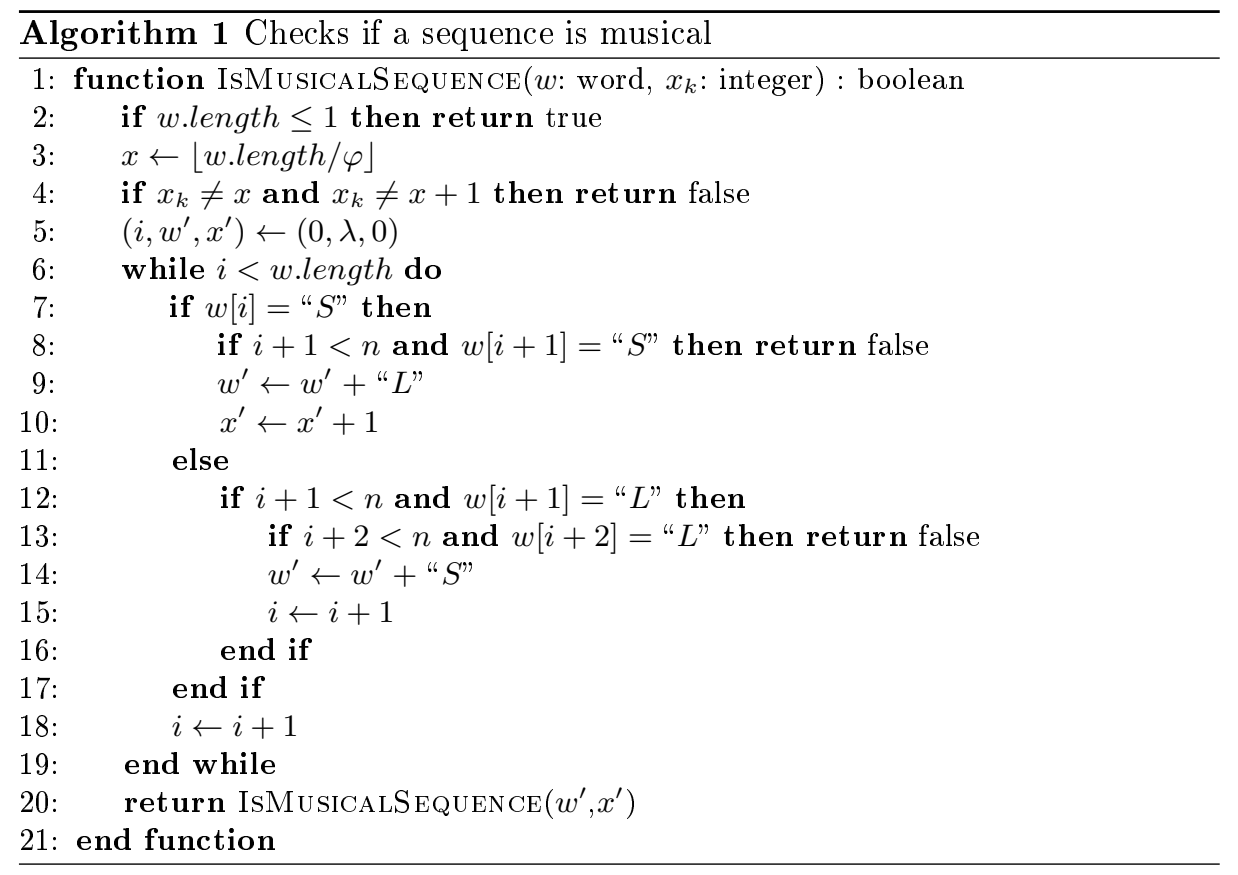

angle makes it easy to identify in which set the bar is. If the representative yields a line that is already in the set, then no new bar is actually added while adding the tile (see next paragraph). Otherwise, the new representative is temporarily inserted at the right place in the array, its distances with its neighbors are computed and the procedure described above is applied to see whether the new word thus obtained is a musical sequence.

To check if two representatives yield the same line, it is sufficient to compute their difference and check if it has the right angle. For this purpose, the following theorem is useful.

Theorem 4. Consider the line $L$ passing through the origin with angle $\theta+\frac{\pi}{2}$, where $\theta \in \mathbb{R}$. Let the basis $B$ consist of unit vectors $\overrightarrow{v_{k}}$ of angles $\theta_{k}=\theta+$ $\frac{k \pi}{5}, k \in\{1,2,3,4\}$ and let $P$ be a Penrose point whose coordinates in $B$ are $\left(a_{0}, a_{1}, a_{2}, a_{3}\right) \in \mathbb{Z}^{4}$. Then

(i) $P$ is on $L$ if and only if $\left(a_{0}, a_{1}, a_{2}, a_{3}\right)$ is a palindrome;

(ii) Points on $L$ are sorted in lexicographical order with respect to their coordinates in $B$

(iii) The ratio $a_{1} / a_{0}$ is an approximation of $\varphi$; in particular, $L$ contains points such that $a_{0}=f_{n}$ and $a_{1}=f_{n+1}$ where $f_{n}$ is the $n^{\text {th }}$ Fibonacci number. 
Proof. (i) $(\Leftarrow)$ If $\left(a_{0}, a_{1}, a_{2}, a_{3}\right)$ is a palindrome then $a_{1}=a_{2}$ and $a_{0}=a_{3}$. Let $z=a \mathrm{e}^{i \theta^{\prime}}$ be the affix of $P$ in the plane. Then

$$
\begin{aligned}
a \mathrm{e}^{i \theta^{\prime}} & =a_{0} \mathrm{e}^{i\left(\theta+\frac{\pi}{5}\right)}+a_{1} \mathrm{e}^{i\left(\theta+\frac{2 \pi}{5}\right)}+a_{1} \mathrm{e}^{i\left(\theta+\frac{3 \pi}{5}\right)}+a_{0} \mathrm{e}^{i\left(\theta+\frac{4 \pi}{5}\right)} \\
& =\mathrm{e}^{i\left(\theta+\frac{\pi}{2}\right)}\left(a_{0} \mathrm{e}^{i \frac{3 \pi}{10}}+a_{1} \mathrm{e}^{i \frac{-\pi}{10}}+a_{1} \mathrm{e}^{i \frac{\pi}{10}}+a_{0} \mathrm{e}^{i \frac{3 \pi}{10}}\right) \\
& =\mathrm{e}^{i\left(\theta+\frac{\pi}{2}\right)}\left(a_{0}\left(\mathrm{e}^{i \frac{-3 \pi}{10}}+\mathrm{e}^{i \frac{3 \pi}{10}}\right)+a_{1}\left(\mathrm{e}^{i \frac{-\pi}{10}}+\mathrm{e}^{i \frac{\pi}{10}}\right)\right) \\
& =\mathrm{e}^{i\left(\theta+\frac{\pi}{2}\right)}\left(2 a_{0} \cos \frac{3 \pi}{10}+2 a_{1} \cos \frac{\pi}{10}\right)
\end{aligned}
$$

where $2 a_{0} \cos \frac{3 \pi}{10}+2 a_{1} \cos \frac{\pi}{10}$ is a real number so $\theta^{\prime}=\theta+\frac{\pi}{2}[\pi]$, thus $P$ is on $L$.

$(\Rightarrow)$ Conversely, if $P$ is on $L$, there exists $a \in \mathbb{R}$ such that $z=a \mathrm{e}^{i\left(\theta+\frac{\pi}{2}\right)}$ is an affix of $P$. Therefore we have

$$
a_{0} \mathrm{e}^{i\left(\theta+\frac{\pi}{5}\right)}+a_{1} \mathrm{e}^{i\left(\theta+\frac{2 \pi}{5}\right)}+a_{2} \mathrm{e}^{i\left(\theta+\frac{3 \pi}{5}\right)}+a_{3} \mathrm{e}^{i\left(\theta+\frac{4 \pi}{5}\right)}=a \mathrm{e}^{i\left(\theta+\frac{\pi}{2}\right)}
$$

We can multiply by $\mathrm{e}^{-i \theta}$ to obtain

$$
a_{0} \mathrm{e}^{i \frac{\pi}{5}}+a_{1} \mathrm{e}^{i \frac{2 \pi}{5}}+a_{2} \mathrm{e}^{i \frac{3 \pi}{5}}+a_{3} \mathrm{e}^{i \frac{4 \pi}{5}}=a \mathrm{e}^{i \frac{\pi}{2}}=a i
$$

Since $\operatorname{Re}(a i)=0$, then we must have

$$
\begin{gathered}
a_{0} \cos \frac{\pi}{5}+a_{1} \cos \frac{2 \pi}{5}+a_{2} \cos \frac{3 \pi}{5}+a_{3} \cos \frac{4 \pi}{5}=0 \\
\Longleftrightarrow\left(a_{0}-a_{3}\right) \cos \frac{\pi}{5}+\left(a_{1}-a_{2}\right) \cos \frac{2 \pi}{5}=0 \\
\Longleftrightarrow\left(a_{0}-a_{3}\right) \cos \frac{\pi}{5}+\left(a_{1}-a_{2}\right)\left(\cos ^{2} \frac{\pi}{5}-\sin ^{2} \frac{\pi}{5}\right)=0 \\
\Longleftrightarrow a_{0}-a_{3}+\left(a_{1}-a_{2}\right)\left(\cos \frac{\pi}{5}-\sin \frac{\pi}{5} \tan \frac{\pi}{5}\right)=0
\end{gathered}
$$

where $\cos \frac{\pi}{5}-\sin \frac{\pi}{5} \tan \frac{\pi}{5}$ is irrational so $a_{1}-a_{2}=0$, i.e. $a_{1}=a_{2}$, and thus $a_{0}=a_{3}$. Hence, $\left(a_{0}, a_{1}, a_{2}, a_{3}\right)$ is a palindrome.

(ii) Note that as a result of equation $3 a=2 a_{0} \sin \frac{\pi}{5}+2 a_{1} \sin \frac{2 \pi}{5}$. Since $\sin \frac{2 \pi}{5}=2 \sin \frac{\pi}{5} \cos \frac{\pi}{5}$ and $\varphi=2 \cos \frac{\pi}{5}$, we have $a=2 \sin \frac{\pi}{5}\left(a_{0}+\varphi a_{1}\right)$. We can see that $a$ is an increasing function in $a_{0}$ and in $a_{1}$, so that $a$ increases as $\left(a_{0}, a_{1}, a_{1}, a_{0}\right)$ increases in lexicographical order.

(iii) Suppose $P^{\prime}$ is represented by $\left(a_{0}+1, a_{1}, a_{1}, a_{0}+1\right)$. If $P, P^{\prime}$ are both in the tiling, it means that they are the points in the concave parts of the darts in a Deuce configuration, thus a kite is forced just above $P^{\prime}$ (due to Ammann bars). Let $P^{\prime \prime}$ be the corner of this kite opposite to $P^{\prime}$, then $P^{\prime \prime}$ is on $L$ and the distance $P^{\prime} P^{\prime \prime}=\varphi$, which means that this time $a_{1}$ has increased by 1 : after $\left(a_{0}+1, a_{1}, a_{1}, a_{0}+1\right)$, the coordinates of the next point on the line have to be $\left(a_{0}+1, a_{1}+1, a_{1}+1, a_{0}+1\right)$. Therefore, $a_{0} \leq a_{1}+1$. Now how often can such a configuration occur on a particular line ? Actually the closest possible 


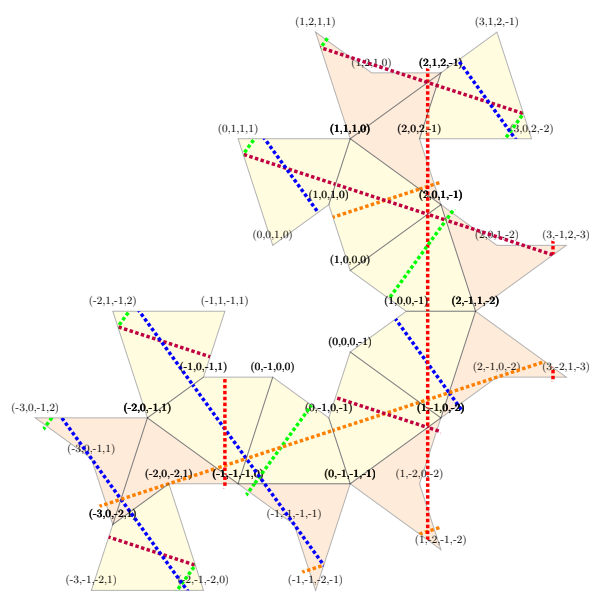

(a) A Penrose tree of size 18

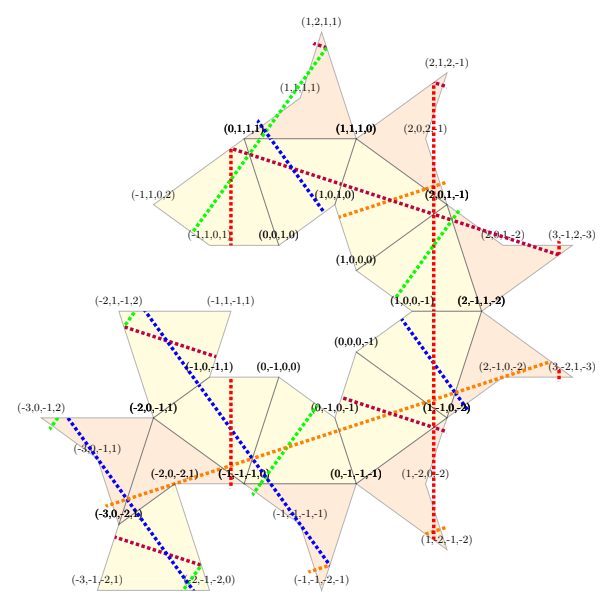

(b) Another Penrose tree of size 18

Fig. 15: Two Penrose trees whose internal tiles have all degree 3.

occurrence yields a short bowtie, and the second shortest a long bowtie. As a result, it means that the points are in a Conway worm and from Proposition 4 , the ratio of long bowties over short ones is an approximation of $\varphi$ (close to the ratio of two consecutive Fibonacci numbers). Considering the local isomorphism property and the placing of Ammann bars from the other sets, proportions are the same on any line containing an arbitrarily large number of Penrose points which are actual vertices of the tiling (we could translate $P$ to the origin). Note that not all such Penrose points appear in the tiling.

Theorem 4 is not really surprising. Indeed, the palindromic structure is due to the fact that this line is the reflection axis of the vectors of the chosen basis when they are placed at the origin (see Figure 12). Therefore, in each basis the points on its symmetry line are palindromes. This gives us a way to easily recognize them: we just have to choose a convenient basis for each Ammann set.

\section{Conclusion}

The problem studied in this article is at the junction between graphs, tilings and words. It is quite intricate due to the non-periodicity of Penrose tilings and of the Fibonacci word. Still, even though we have not been able to find the leaf function for Penrose tilings yet, we managed to restrict the range of possible values. The gain in efficiency granted by the discrete representation, and the procedure described in Section 7 will certainly allow us to exhibit some interesting induced subtrees. We show in Figure 15 two Penrose trees of size 18, yielded by our current algorithm. In the future, we intend to improve its performance so that it can prove our conjecture that there is no Penrose tree of size greater than 18 whose internal tiles are all of degree 3.

We also have good hope that we can find $L_{k d}$ or at least finer bounds thanks to a particular patch which has more leaves than a Penrose caterpillar of the same order constructed as described in Section 5. If the leaf function for kites 
and darts tilings can be found, a similar procedure could be pursued in order to find the analogous function for Penrose tilings by rhombs, and possibly for other aperiodic tilings which share similar properties.

\section{References}

1. Allouche, J.P., Shallit, J.O.: Automatic sequences : theory, applications, generalizations. Cambridge University Press, Cambridge, UK (2003)

2. Blondin Massé, A., de Carufel, J., Goupil, A.: Saturated Fully Leafed Tree-Like Polyforms and Polycubes. ArXiv e-prints (Mar 2018), https://arxiv.org/abs/ 1803.09181v1

3. Blondin Massé, A., de Carufel, J., Goupil, A., Lapointe, M., Nadeau, É., Vandomme, É.: Leaf realization problem, caterpillar graphs and prefix normal words. ArXiv e-prints (Dec 2017), https://arxiv.org/abs/1712.01942

4. Blondin Massé, A., de Carufel, J., Goupil, A., Lapointe, M., Nadeau, É., Vandomme, É.: Fully leafed induced subtrees. In: Iliopoulos, C., Leong, H.W., Sung, W.K. (eds.) Combinatorial Algorithms. pp. 90-101. Springer International Publishing, Cham (2018). https://doi.org/10.1007/978-3-319-94667-2_8

5. Blondin Massé, A., de Carufel, J., Goupil, A., Samson, M.: Fully leafed tree-like polyominoes and polycubes. In: Brankovic, L., Ryan, J., Smyth, W.F. (eds.) Combinatorial Algorithms. pp. 206-218. Springer International Publishing, Cham (2018). https://doi.org/10.1007/978-3-319-78825-8_17

6. de Bruijn, N.G.: "Algebraic theory of Penrose's non-periodic tilings of the plane". Mathematics Proceedings A84, 39-66 (1981), reprinted in [17]

7. Burcsi, P., Fici, G., Lipták, Z., Ruskey, F., Sawada, J.: On prefix normal words and prefix normal forms. Theoretical Computer Science 659, 1 13 (2017). https://doi.org/https://doi.org/10.1016/j.tcs.2016.10.015 http://www. sciencedirect.com/science/article/pii/S0304397516305680

8. Gardner, M.: Penrose Tiles to Trapdoor Ciphers. The Mathematical Association of America, revised edn. (1997), iSBN 0-883856-521-6

9. Grünbaum, B., Shephard, G.C.: Tilings and Patterns. W. H. Freeman and Company, New York (1987), iSBN 0-7167-1193-1

10. Grünbaum, B., Shephard, G.C.: Tilings and Patterns. Dover Publications, Inc. (2016), second edition. ISBN 978-0-486-46981-2

11. Harary, F., Schwenk, A.J.: The number of caterpillars. Discrete Mathematics 6(4), 359 - 365 (1973). https://doi.org/https://doi.org/10.1016/0012-365X(73)90067-8. http://www.sciencedirect.com/science/article/pii/0012365X73900678

12. Penrose, R.: The Rôle of Aesthetics in Pure and Applied Mathematical Research. Bulletin of the Institute of Mathematics and its Applications pp. 266-271 (1974), reprinted in [17]

13. Penrose, R.: Pentaplexity. Math. Intelligencer 2(1), 32-37 (1978)

14. Penrose, R.: Set of tiles for covering a surface. United States Patent $(4,133,152)$ (1979), jan. 9, 1979

15. Porrier, C., Blondin Massé, A.: The leaf function for graphs associated with penrose tilings. In: 2019 International Conference on Graph Computing (GC-2019). IEEE Computer Society Press digital library (2019)

16. Reinsch, M.W.: Lattice representations of Penrose tilings of the plane. arXiv eprints math-ph/9911024 (Nov 1999)

17. Steinhardt, P.J., Ostlund, S.: The Physics of Quasicrystals. World Scientific (1987), collection of reprints. ISBN 9971-50-226-7 